J. Clin. Chem. Clin. Biochem.

Vol. 14, 1976, pp. 73-77.

\title{
Gaschromatographische Bestimmung von Ameisensäure im Harn in Form von Kohlenmonoxid
}

\author{
Von J. Angerer \\ Aus dem Zentralinstitut für Arbeitsmedizin (Direktor: Prof. Dr. med. G. Lehnert) der Freien und Hansestadt
Hamburg
}

(Eingegangen am 15. Juli/5. November 1975)

Zusammenfassung: Es wird eine gaschromatographische Methode zur Bestimmung der Ameisensäurekonzentration im menschlichen Harn beschrieben. Die analytische Zuverlässigkeit der Methode erfüllt die Anforderungen der statistischen Qualitätskontrolle. Die Wiederauffindungsraten lagen zwischen 101,2 und 105,7\%; die Variationskoeffizienten zwischen 2,9 und 7,2\%. Die Spezifität der Methode erwies sich bei der Analyse einer Reihe anderer im Harn vorkommender Stoffe, die keine Beeinträchtigung der Ameisensäurebestimmung hervorriefen. Die Nachweisgrenze von rund 4,3 $\mu \mathrm{mol} / 1$ Ameisensäure im Harn ermöglicht es, die Ameisensäurekonzentration in Harn von Normalpersonen zu bestimmen. Bei einem Kollektiv $(n=30)$ von Normalpersonen wurden Ameisensäureausscheidungen zwischen 0 und 2,79 mmol/Lund im Mittel 0,39 $\pm 0,60 \mathrm{mmol} / \mathrm{l}$ gemessen.

\section{Gas chromatographic determination of formic acid in urine as carbon monoxide}

Summary: A gas chromatographic, method for determining formic acid in human urine is described. The analytical reliability of this method fullfills the criteria of statistical quality control. The rate of recovery is 101.2 to $105.7 \%$, the variability coefficients lie between 2.9 and $7.2 \%$. The selectivity of this method is demonstrated by analysing a group of components normally occuring in urine which did not interfere with the determination of formic acid. The detection limit of about $4.3 \mu \mathrm{mol} / 1$ formic acid in urine permits the determination of the concentration of formic acid in the urine of normal persons. The concentrations of formic acid in the urine of a group of normal persons lies between 0 and $2.79 \mathrm{mmol} / 1$. The average concentration was $0.39 \pm 0.60 \mathrm{mmol} / \mathrm{l}$.

\section{Eịnleitung}

Nach gegenwärtigen Kenntnissen darf davon ausgegangen werden, daß die industriell in großem Maße verwendeten Methylhalogenide, Methylchlorid (1) und Methylbromid (2), im Körper zu den entsprechenden Halogenwasserstoffen und Methanol $(3,4,5)$ hydrolysiert werden. Letzteres wird seinerseits über Form: aldehyd zu Ameisensäure metabolisiert und im Harn ausgeschieden. Der Bestimmung der Ameisensäure im Harn könnte damit als Beurteilungsgrundlage für das Ausmaß der Giftstoffbelastung arbeitsmedizinische Relevanz zukommen. Eine zuverlässige und praktische Bestimmungsmethode ișt jedoch nicht nur unter präventiv-medizinischen Aspekten von Interesse, sondern könnte auch zu einer Vertiefung der bisher lückenhaften Kenntnisse über den Metabolismus der Methylhalogenide führen.

Unter diesem Gesichtspunkt wird im folgenden ein gas: chromatographisches Verfahren zur Bestimmung der Ameisensäure im Harn vorgestellt, das sich durch Prạk= tikabilität sowie Validität auszeichnet uño unter gaschromạtographischen Bedingungen arbeitet, die auch zur
Bestimmung von Kohlenmonoxid im Blut und in der Luft Verwendung finden können (6). Auf die Darstellung der bisher bekannten Bestimmungsmethoden für Ameisensäure soll hier verzichtet werden. Hier sei auf die umfangreichen Literaturhinweise in den Arbeiten von Rietbrock (7) und Doms (8) verwiesen.

\section{Methodik \\ Prinżip der Methode}

Die im Harn vorliegende Ameisensäure wird durch Wasserdampfdestillation von anderen Bestandteilen abgetrennt. Als einzige aliphatische Carbonsäure zerfält sie unter der Einwirkung von konzentrierter Schwefelsäure zu Wasser und Kohlenmonoxid. Dieses wird nach katalytischer Reduktion zu Methan durch eine gaschromatographische Analyse eines aliquoten Teils der Dampfphase quantitativ mit einem Flammenionisationsdetektor bestimmt.

Experimentelles

Aufarbeitung des Harns

$20 \mathrm{ml}$ Harn werden mit Schwefelsäure $(106,6 \mathrm{~g} / \mathrm{kg})$ auf pH 2 eingestellt und einer Wasserdampfdestillation unterworfen. In einem $100 \mathrm{ml}$ Meßkölbchen, in dem $5 \mathrm{ml} 5 \mathrm{~mol} / \mathrm{l}$ Natronlauge vorgelegt sind, werden genau $95 \mathrm{ml}$ Destillat aufgefangen. Da- 
nach schüttelt man den Meßkolben mehrmals um und bewahrt das Destillat bis zur gaschromatographischen Analyse im Kühlschrank auf. Grundsätzlich werden bei jeder Analysenserie zwei Reagenzienleerwerte mitgeführt, die in gleicher Weise aufgearbeitet werden.

\section{Erzeugung von Kohlenmonoxid}

Zur Erzeugung von Kohlenmonoxid aus den ameisensäurehaltigen Lösungen werden $2 \mathrm{ml}$ des Wasserdampfdestillates in eine mit Stickstoff gespülte Stechampulle (20 ml; Perkin Elmer) pipettiert. Um bei der weiteren Aufarbeitung die Wärmereaktion und damit mögliche Kohlenmonoxidverluste möglichst gering zu halten, werden die Proben nun in der Tiefkühltruhe eingefroren (30 Minuten). Danach fügt man $15 \mathrm{ml} 95 \%$ iger Schwefelsäure p. a. (Merck Nr. 731) zu und verschließt die Stechampulle sofort. Unter vorsichtigem Umschwenken wird die Probe nun vollständig gemischt, verschlossen und über Nacht (18 Stunden) bei $35^{\circ} \mathrm{C}$ in einen Wärmeschrank gestellt.

\section{Gaschromatographische Analyse}

Mittels einer gasdichten Spritze $(250 \mu$ l Hamilton) entnimmt man $200 \mu l$ aus der Gasphase (head space) der Stechampulle und injiziert in den entsprechend modifizierten Gaschromatographen (Abb. 1). Die Arbeitsbedingungen für die gaschromatographische Analyse entsprechen der von uns beschriebenen $\mathrm{CO}-$ Bestimmungsmethode (6) des aus Ameisensäure gebildeten Kohlenmonoxids, das auf einer Nickelkatalysatorstrecke (Abb. 1) zu Methan reduziert wird. Die gaschromatographischen Bedingungen sind in Tabelle 1 zusammengefaßt.

\section{Kalibrierung}

Zur Erstellung von Standardkurven werden wäßrige Lösungen von Natriumformiat hergestellt, die $z$ wischen 0,11 und $2,17 \mathrm{mmol} / 1$ Ameisensäure enthalten, und wie Harnproben aufgearbeitet.

\section{Berechnung}

Zur Berechnung der Analysenergebnisse wird von den Flächen der Probenpeaks der jeweilige mittlere Reagenzienleerwert ab-

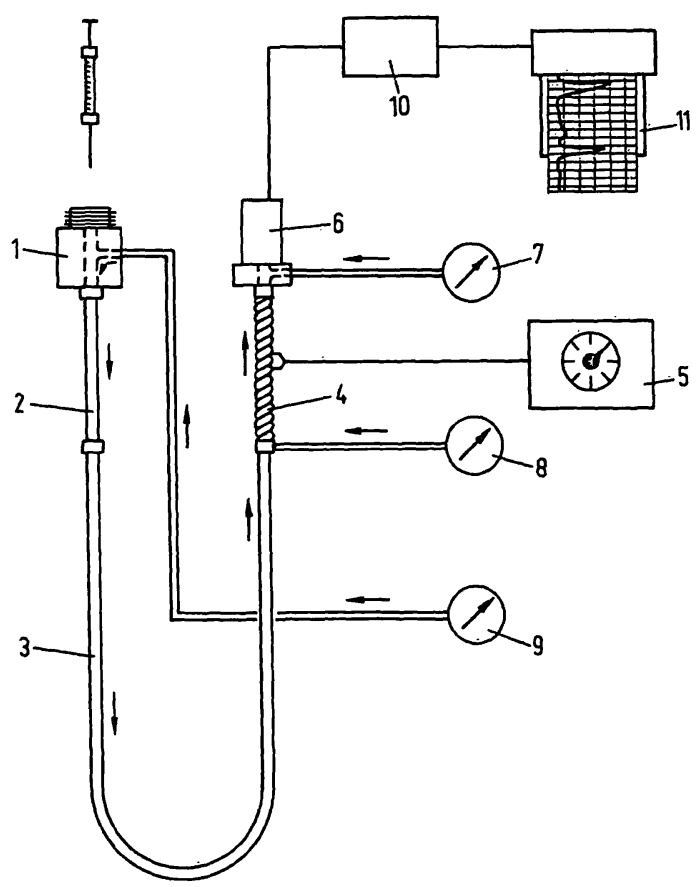

Abb. 1. Schematische Darstellung der gaschromatographischen Ameisensäure (Kohlenmonoxid) Bestimmung. (1 Injektor; 2 Vorsäule; 3 Trennsäule; 4 Kataly satorsäule mit Heizbandage; 5 Regeltrafo; 6 FID; 7 synthetische Luft; 8 Wasserstoff; 9 Stickstoff; 10 Verstärker 11 Schreiber).
Tab. 1. Gaschromatographische Arbeitsbedingungen.

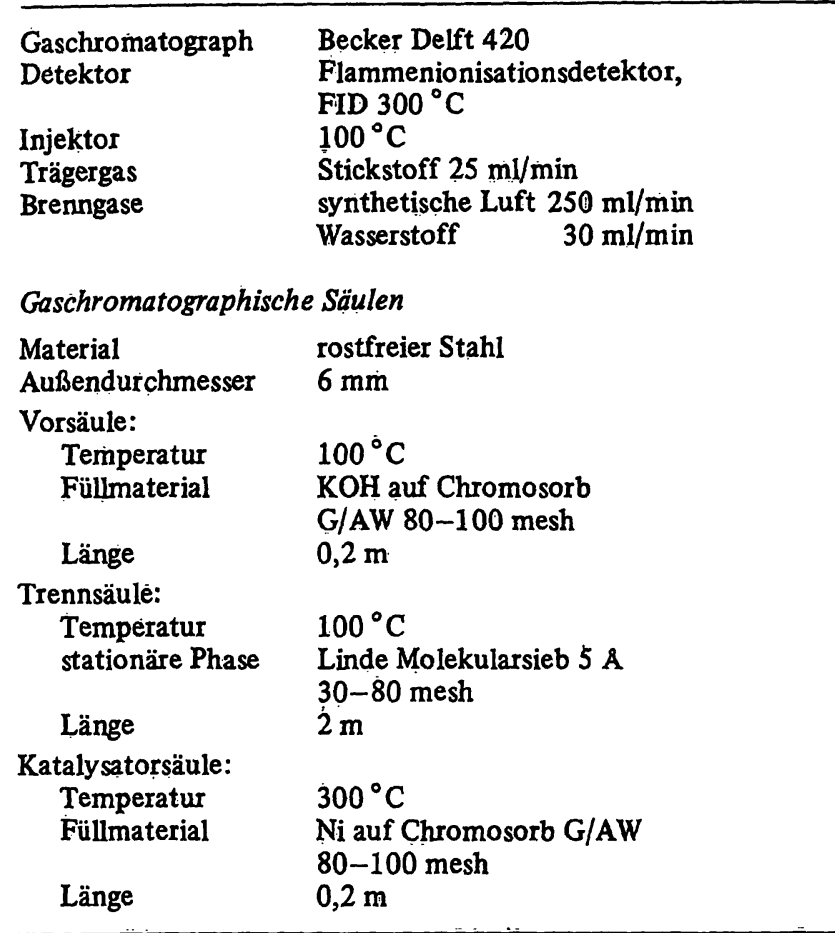

gezogen. Die erhaltenen Werte werden durch die Fläche eines bei jeder Analysenserie mit analysierten CO-Standards (Linde Plastigas $100 \mathrm{vpm} \triangleq 1 \mathrm{~cm}^{3} / \mathrm{m}^{3}$ CO Stickstoff) dividiert. Mit diesen Quotienten geht man in die entsprechende Standardkurve ein und erhält die Konzentration der Amẹeisensäure in $\mathrm{mmol} / \mathrm{l}$.

\section{Ërgebnisse}

Aus Abbildung 2 geht hervor, daß die unter Einwirkung der Schwefelsäure ablaufende Bildung von Kohlenmonoxid aus Ameisensäure nach ca 8 Stunden abgeschlossen ist. Der Meßwert bleibt über ca 27 Stunden konstant.

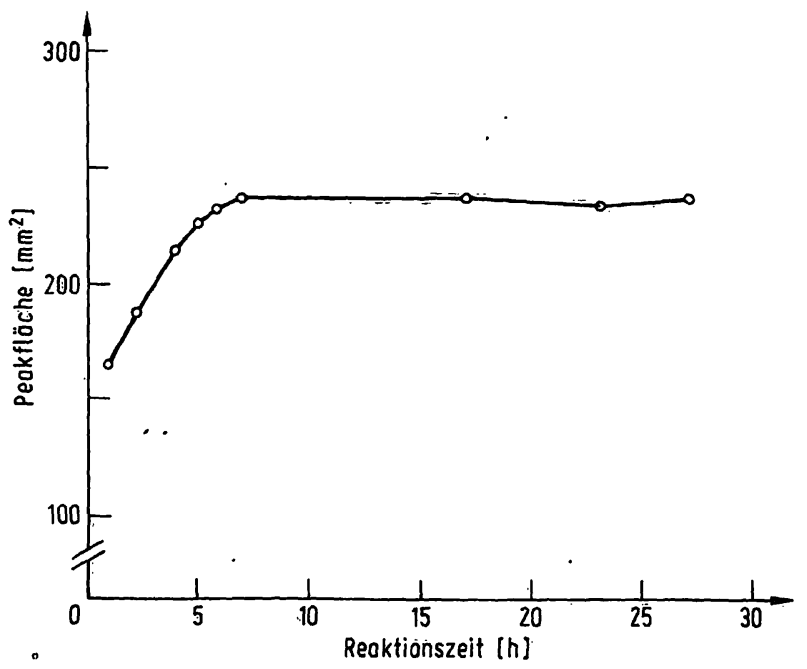

Abb. 2. Einfluß der Einwirkungszeit von konzentrierter Schwefelsäure auf die gemessenen Peakflächen vọn Ameisensäurelösungen. 
Die Retentionszeit für das zur Messung kommende Kohlenmonoxid beträgt 8,8 Minuten (Abb. 3).

Die Standardkurve, bei der der Quotient aus den Peakflächen des freigesetzten Kohlenmonoxids und der Peakfläche eines während der gleichen Analysenserie mitgemessenen CO-Kalibriergases gegen die eingesetzten Ameisensäurekonzentrationen aufgetragen wurde, erwies sich im untersuchten Bereich (0,11-2,17 mmol/1 Ameisensäure $)$ als linear $(y=0,0204+0,2796 x ; r=$ 0,9997). Zur Uberprüfung der Selektivität des Analysenverfahrens wurde eine Reihe von normalerweise im Harn vorkommenden Substanzen (Tab. 2) hinsichtlich möglicher Störeinflüsse untersucht. Dazu wurden wäßrige Lösungen dieser Stoffe in einer Konzentration von je $1 \mathrm{~g} / \mathrm{l}$ dem Analysengang unterworfen. Es zeigte sich, daß diese Substanzen, die unter dem Einfluß von konzentrierter Schwefelsäure ebenfalls $\mathrm{CO}$ abspalten, wie z. B. die 2-Hydroxy- und die 2-Oxocarbonsäuren, durch die Wasserdampfdestillation abgetrennt werden konnten bzw. nicht störend in Erscheinung traten. Die Genauigkeit und Richtigkeit der Methode wurde durch mehrmalige Analyse von Harnproben getestet,

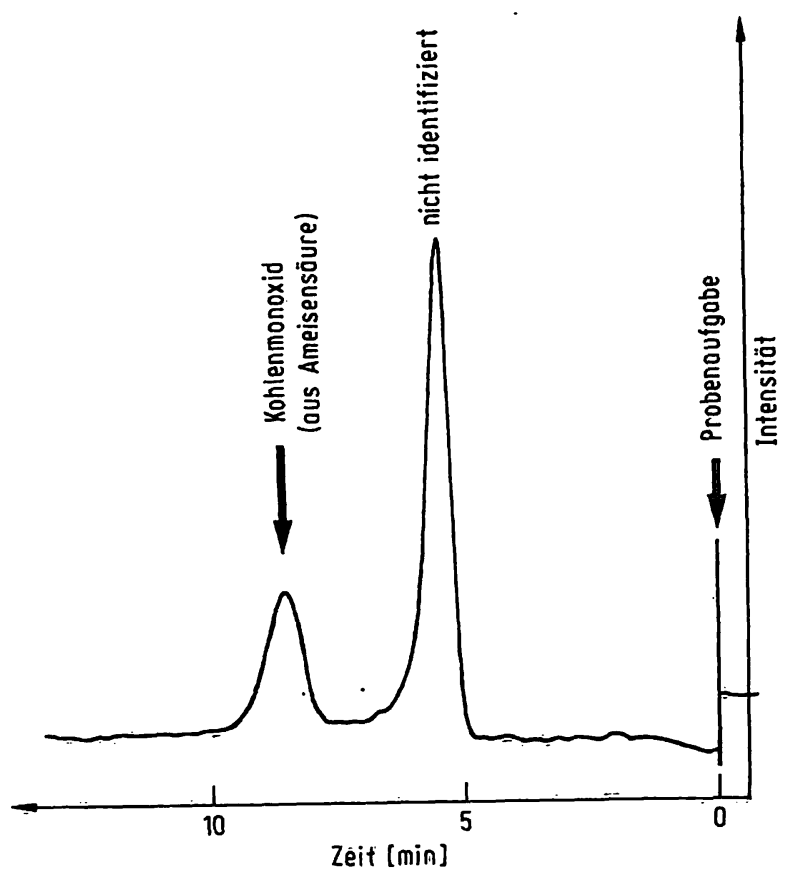

Abb. 3. Gaschromatogramm ènèr aufgẹarbeiteten Harnprobe zur Bestimmung von Ameisensäure. Die Ameisensäurekonzentration betrug $0,402 \mathrm{mmol} / \mathrm{l}$.

Tab. 2. Stoffe, dịe hinsichtlich möglicher Störeffekte bei der Ameisensäurebestimmung untersucht wurden. Die Konzentration der untersuchten Lösungen betrug jeweils $1 \mathrm{~g} / \mathrm{i}$.

Äpfelsäure

Brenztraubensäure

Citronensäure

Formiminoglutaminsă̈ure
Glyoxylsäure

2-Oxoglutarsäure

Milchsäure

Oxalsäure denen unterschiedliche Ameisensäuremengen zugesetzt

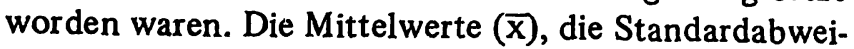
chungen (s) und die Variationskoeffizienten (V) dieser Bestimmungen sowie die unter Berücksichtigung des Harnleerwertes errechneten Wiederauffindungsraten sind in Tabelle 3 zusammengefaßt.

Die Nachweisgrenze der Ameisensäure in Form von Kohlenmonoxid beträgt unter Zugrundelegung eines SignalRauschverhältnisses von $3: 14,3 \mu \mathrm{mol} / 1$ Ameisensäure im Harn.

Zur Uberprüfung der praktischen Anwendbarkeit sowie zur Bestimmung der normalerweise im Urin vorkommenden Ameisensäurekonzentration wurde bei einem Kollektiv von 30 Büroangestellten der Ameisensäuregehalt des Harnes bestimmt. Gaschromatographisch wurden Ameisensäurekonzentrationen zwischen 0 und $2,79 \mathrm{mmol} / 1, \mathrm{im}$ Mittel 0,39 $\pm 0,60 \mathrm{mmol} / 1$, gemessen. Zusätzlich wurde bei dem gleichen Kollektiv die Ameisensäure photometrisch nach Rietbrock (10) bestimmt. Hier ergaben sich Ameisensäureausscheidungen zwischen 0 und 4,24 mmol/1. Die mittlere Ausscheidung betrug in diesem Fall 0,56 $\pm 0,89 \mathrm{mmol} / 1$. Die nach beiden Methoden bestimmten individuellen Ameisensäurekonzentrationen im Harn sind in Tabelle 4 zusammengestellt. Die nach beiden Methoden erhaltenen mittleren Ameisensäurekonzentrationen unterscheiden sich in einem t-Test mit verbundenen Stichproben statistisch signifikant $\left.\left(t=2,614^{*}\right)^{1}\right)$.

Bei dem gleichen Kollektiv korrelieren die photometrisch und die gaschromatographisch ermittelten Ameisensäurekonzentrationen straff $(y=0,0077+$ $\left.1,4168 \mathrm{x} ; \mathrm{r}=0,958^{* * *}\right)^{1}$ ). Die nach beiden Methoden bestimmten individuellen Ameisensäurekonzentrationen im Harn sind in Tabelle 4 zusammengestellt.

\section{Diskussion}

In Folge der Sonderstellung der Ameisensäure unter den flüchtigen aliphatischen Carbonsäuren war ihre gaschromatographische Bestimmung, wie Doms (8) mitteilt, unter anderem wegen des damit verbundenen geringeren Nachweisvermögens problematisch. Ausreichende Nachweisgrenzen konnten nur nach vorhergehen-

Tab. 3. Genauigkeit und Richtigkeit von Ameisensäureanalysen im Harn.

\begin{tabular}{|c|c|c|c|c|c|}
\hline \multirow[t]{3}{*}{$\mathbf{n}$} & \multirow{3}{*}{$\begin{array}{l}\text { Sollwert } \\
\text { (Leerwert + } \\
\text { zugesetzte } \\
\text { Menge } \\
\text { [mmol/1]) }\end{array}$} & \multicolumn{3}{|l|}{ Istwert } & \multirow{3}{*}{$\begin{array}{l}\text { Wieder- } \\
\text { auf- } \\
\text { findung } \\
\%\end{array}$} \\
\hline & & $\overline{\overline{\mathbf{x}}}$ & $\mathbf{s}$ & $\mathbf{V}$ & \\
\hline & & [mmol/1] & [mmol/1] & [\%] & \\
\hline $\begin{array}{l}6 \\
6 \\
6\end{array}$ & $\begin{array}{l}0,206+0 \\
0,206+0,109 \\
0,209+1,086\end{array}$ & $\begin{array}{l}0,206 \\
0,333 \\
1,309\end{array}$ & $\begin{array}{l}0,006 \\
0,024 \\
0,091\end{array}$ & $\begin{array}{l}2,9 \\
7,2 \\
7,0\end{array}$ & $\begin{array}{l}- \\
105,7 \\
101,1\end{array}$ \\
\hline
\end{tabular}

1) *: $\mathrm{p}<0,05 ; * * *$ : $<<0,001$. 
Tab. 4. Gemessene Ameisensäurekonzentrationen in Harnproben eines Normalkollektivs $(n=30)$.

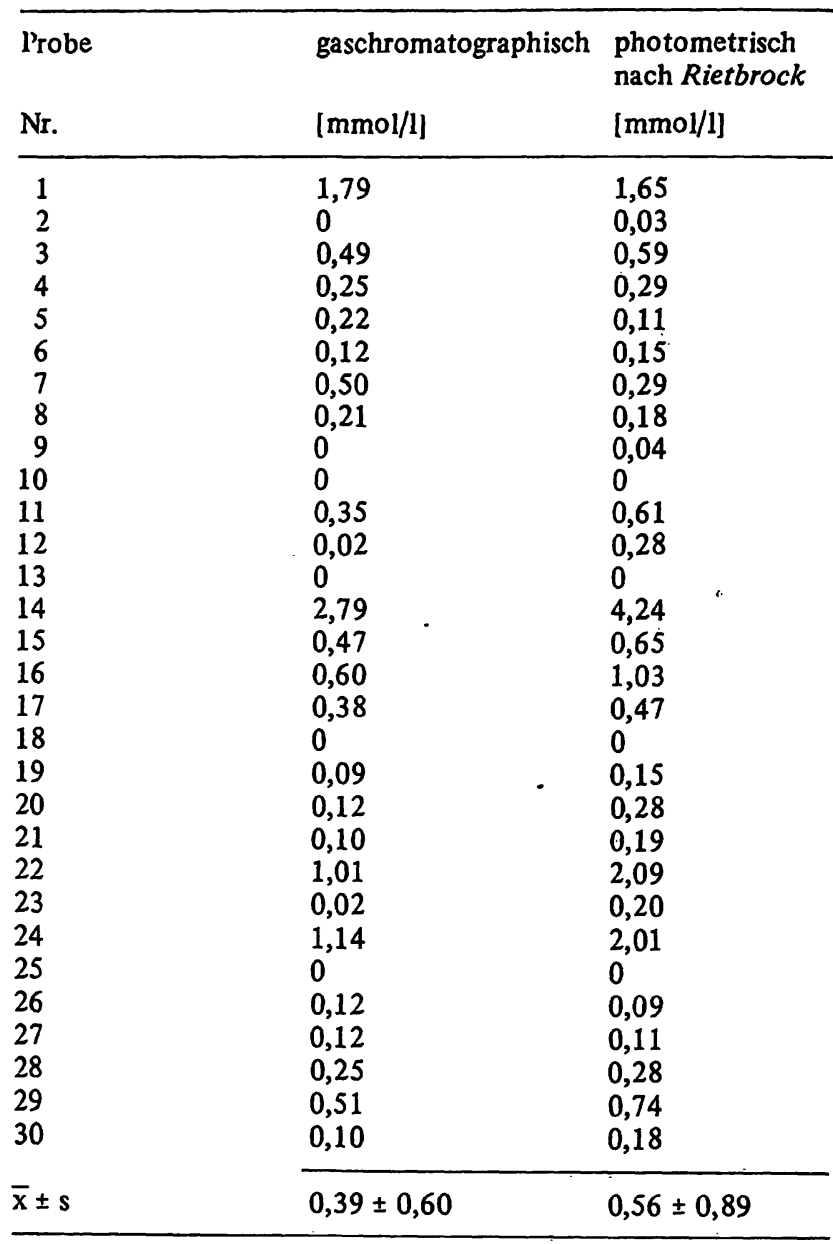

der Veresterung erzielt werden. Die bei unserer Methode an Stelle der Veresterung durchgeführte Spaltung der Ameisensäure ist - bei zumindest ebenbürtiger Empfindlichkeit - einfacher durchzuführen und damit weniger störanfällig. Die zur Abtrennung der 2-Oxo- bzw. 2-Hydroxycarbonsäuren, sowie anderer störender Substanzen (Tab. 2) notwendige Wasserdampfdestillation stellt allerdings einen gewissen Arbeitsaufwand dar. Dieser kann jedoch durch eine routinemäßige Durchführung dieser unproblematischen Operation stark reduziert werden, so daß von einer technischen Assistenz täglich bis zu 20 Harnproben analysiert werden können. Darüber hinaus bietet dieses Verfahren die Möglichkeit ohne Änderung der gaschromatographischen Parameter Kohlenmonoxid in Blut und Luft zu bestimmen, was besonders im arbeitsmedizinisch-toxikologischen Labor eine sinnvolle Auslastung der Gerätekapazität zuläßt.

Die erwiesene Spezifität (s. Abb. 3 und Tab. 2) des Analysenverfahrens beruht auf dem charakteristischen
Zerfall der Ameisensäure in Wasser und Kohlenmonoxid unter der Einwirkung von konzentrierter Schwefelsäure. Andere Harnbestandteile, insbesondere 2-Oxo- und 2-Hydroxycarbonsäuren, die unter diesen Bedingungen ebenfalls $\mathrm{CO}$ abspalten, werden durch eine Wasserdampfdestillation abgetrennt. Weitere Substanzen, wie Kohlendioxid, das wie Kohlenmonoxid ebenfalls katalytisch zu Methan reduziert wird, werden gaschromatographisch auf der Molekularsiebsäule abgetrennt. Durch die Vèrwendung von $\mathrm{KOH}$ in der Vorsäule werden weitere Verunreinigungen zurückgehalten, so daß auch im monatelangen Betrieb kein Unwirksamwerden der Katalysatorstrecke zu beobachten war. Der Peak, der auch bei der Analyse von Reagenzienblindwerten bei einer Retentionszeit von 5,4 min auftritt, konnte bisher nicht identifiziert werden.

Die Genauigkeit und Richtigkeit der Methode ist bei Variationskoeffizienten um $7 \%$ und Wiederauffindungsraten zwischen 101 und $105 \%$ als gut zu bezeichnen und erfüllt die Anforderungen der statistischen Qualitätskontrolle $(9,10)$. Diese Ergebnisse sind auf die hervorragende gaschromatographische Abtrennung, sowie auf die Verwendung eines externen Kohlenmonoxid-Kalibriergas-Standards zurückzuführen. $\mathrm{Da}$, wie die Qualitätskontrolle zeigt, der von Tag zu Tag auftretende Analysenfehler $10 \%$ nicht übersteigt, konnte auf die Verwendung eines inneren Standards verzichtet werden.

Die Nachweisgrenze von $4,3 \mu \mathrm{mol} / 1$, die auf die Verwendung eines Flammenionisationsdetektors zurückzuführen ist, erlaubt es, den Ameisensäuregehalt auch im Harn von Normalpersonen festzustellen. Die hier im Vergleich zur photometrischen Methode nach Rietbrock gemessenen niedrigeren Ameisensäureausscheidungen, dürften hauptsächlich auf die größere Spezifität der gaschromatographischen Methode zurückzuführen sein.

Welche Bedeutung dem Nachweis der Ameisensäure als Uberwachungsuntersuchung zur Beurteilung von Methylhalogenid bżw. Methanolexpositionen zukommt, muß̧ weiteren Untersuchungen vorbehalten bleiben. Es kann jedoch schon heute gesagt werden, daß die Methode aufgrund ihrer hohen analytischen Zuverlässigkeit sowie ihrer Praktikabilität die Voraussetzung für eine weitere arbeitsmedizinische Erkenntnisgewinnung auf dem Gebiet des Methylhalogenid-Metabolismus schafft. Außerdem dürfte das Verfahren sicherlich auch z. B. in der Lebensmittelchemie zur quantitativen Bestimmung des Konservierungsmittels Ameisensäure sinnvoll einzusetzen sein.

\section{Danksagung}

Ich bedanke mich bei Frau $I$. Wendländer und Herrn T. Alturayhy für die technische Assistenz bei der Erarbeitung dieser Methode. 


\section{Literatur}

1. Browning, E. (1965), Toxicity and metabolism of industrial solvents, S. 230-231, 312, Elsevier, Amstcrdam.

2. Encyclopaedia of Occupational Health and Safety. (1971), $I$, 213-214, International Labour Office, Genf

3. Flury, F. \& Wirth, W. (1937), Arch. Gewerbepathol. Ge werbehyg. 7, 221-226.

4. Soucek, B. (1961), Arch. Gewerbepathol. Gewerbehyg. 18, $370-383$.

5. Sujbert, L. (1967), Arch. Toxikol. 22, 233-235.

6. Mundt, W., Ruppel, R., Kassebart, V., Angerer, J. \& Szadkowski, D. (1974), Inn. Med. 3, 145-151.
7. Rietbrock, N. \& Hinrichs, W.-D. (1964), Klin. Wochenschr. $19,981-985$.

8. Doms, E. K. (1975), J. Chromatogr. 105, 79-88.

9. Richtlinien der Bundesärztekammer zur Durchführung von Maßnahmen der statistischen Qualitätskontrolle und von Ringversuchen in der Heilkunde (1971), Deutsches Ärzteblatt $68,2228-2231$

10. Lehnert, G. \& Szadkowski, D. (1974), Die Berufsgenossenschaft $26,422-424$.
Dr. rer. nat. J. Angerer

Zentralinstitut für Arbeitsmedizin der Freien und Hansestadt Hamburg

2 Hamburg 76

Adolph-Schönfelder-Straße 5

Postfach 4556 
